\title{
Influence on the Performances of Foamed Concrete by Silica Aerogels
}

\author{
Cheng Hai-li ${ }^{1}$, Yang Fei-hua ${ }^{2}$, Wang $\mathrm{Yi}^{3}$, Hao Li-wei ${ }^{2}$ \\ ${ }^{1}$ College of Civil Engineering, North China University of Technology, Beijing, China \\ ${ }^{2}$ State Key Laboratory of Solid Waste Reuse for Building Materials (Beijing Building Materials Academy of Sciences Research), Beijing, \\ China \\ ${ }^{3}$ State Grid Yuyao Power Supply Company, Yuyao, China
}

\section{Email address:}

tkggig@sina.com (Cheng Hai-li), chyangfeihua@126.com (Yang Fei-hua),wang_yi@vip.126.com (Wang Yi), hlw717@126.com (Hao Li-wei)

\section{To cite this article:}

Cheng Hai-li, Yang Fei-hua, Wang Yi, Hao Li-wei. Influence on the Performances of Foamed Concrete by Silica Aerogels. American Journal of Civil Engineering. Vol. 3, No. 5, 2015, pp. 183-188. doi: 10.11648/j.ajce.20150305.18

\begin{abstract}
Aiming at the problems of organic thermal insulation material, $\mathrm{SiO}_{2}$ aerogel was mixed into foamed concrete in two different ways, to investigate its effects on thermal conductivity, compression strength and volume water absorption of foamed concrete. XRD, FT-IR and SEM have also been used to analyze the relationship between both the amount and the mixing way of aerogel and macro properties of foamed concrete. The results show that directly mixing aerogel into foamed concrete has limited effects on improving its performace, mixing $0.5 \%$ of aerogal has little effect on improving thermal conductivity, while mixing $2.5 \%$ of aerogel can greatly decrease compression strength though thermal conductivity has been improved. If aerogal has been premixed with glycol and hydroxypropyl methyl cellulose(HPMC) ether and then mixed into foamed concrete, the compression strength will decrease less while thermal conductivity could decrease by $35 \%$, volume water absorption by $31 \%$.
\end{abstract}

Keywords: $\mathrm{SiO}_{2}$ Aerogel, Foamed Concrete, Thermal Conductivity, Compression Strength, Volume Water Absorption

\section{$\mathrm{Si}_{2}$ 气凝胶对发泡混凝土性能影响研究}

程海丽 ${ }^{1}$, 杨飞华 ${ }^{2}$, 王翌 ${ }^{3}$, 郝利炜

${ }^{1}$ 北方工业大学土木工程学院, 北京, 中国

${ }^{2}$ 固废资源化利用与节能建材国家重点实验室（北京建筑材料科学研究总院有限公司）, 北京, 中国

${ }^{3}$ 国网渐江余姚市供电公司，余姚，中国

\section{邮箱}

tkggig@sina.com（程海丽）, chyangfeihua@126.com（杨飞华）,wang_yi@vip. 126.com（王翌）, h1w717@126. com（郝利炜）

摘要：针对目前有机绝热材料存在的问题, 将 $\mathrm{SiO}_{2}$ 气凝胶以两种不同的方式掺入发泡混凝土中, 研究其对发泡混凝土 导热系数、抗压强度及体积吸水率的影响，并用XRD，FT-IR和SEM的手段分析了气凝胶掺量及掺入方式与宏观性能的关 系。结果表明: 气凝胶直接掺入发泡混凝土中对其性能的改善作用有限，掺量0.5\%时导热系数改善效果甚微，掺量 $2.5 \%$ 时导热系数虽有改善但抗压强度下降程度较大, 而将气凝胶与乙二醇和羟丙基甲基纤维素醚预混后掺入发泡混凝土中, 在同样掺量时可使其强度下降较小而导热系数降低 $35 \%$ 、体积吸水率降低 $31 \%$ 。

关键词: $\mathrm{SiO}_{2}$ 气凝胶, 发泡混凝土, 导热系数, 抗压强度, 体积吸水率 


\section{1. 引言}

随着国民经济的发展, 建筑能耗已占据社会总能耗的 $1 / 4$, 建筑节能已成为刻不容缓的艰巨任务。有机绝热材 料 (主要包括聚苯板 (EPS)、挤塑板 (XPS) 和聚氨酯 (PU) 三种) 以其质轻、㓞性高、绝热性好等优点广泛应用于建 筑节能领域, 然而, 近年来全国各地建筑火灾频发, 使其 易燃、防火安全性差的缺陷再次成为政府监管部门和相关 行业关注的焦点。传统的发泡混凝土作为无机材料, 因其 不燃、不老化、火灾时不产生有毒气体等优点成为替代有 机绝热材料的选择之一, 也成为近年来的研究热点。虽然 目前发泡混凝土的导热系数可低至 $0.08 \mathrm{~W} /(\mathrm{K} \cdot \mathrm{m})[1]$, 但与目前常用的有机绝热材料相比 (导热系数一般在 $0.024-0.041 \mathrm{~W} /(\mathrm{K} \cdot \mathrm{m})$ 之间) [2-3], 其导热系数仍较 大, 不能满足保温节能的要求。Si02气凝胶 (以下简称气 凝胶）是一种纳米多孔材料，其内部孔洞率可高达 $80 \%$ 以 上, 导热系数可低至 $0.015 \mathrm{~W} /(\mathrm{K} \cdot \mathrm{m})$, 是一种性能优异 的绝热材料。将气凝胶应用于发泡混凝土中以改善其绝热 性能的研究文献目前尚不多见, 因此, 本文以导热系数为 $0.079 \mathrm{~W} /(\mathrm{K} \cdot \mathrm{m})$ 的发泡混凝土为基础, 研究气凝胶的掺 入对其导热系数、抗压强度及体积吸水率的影响。

\section{2. 主要原材料及其性能}

表1 P. 0 42.5水泥的主要性能。

\begin{tabular}{llllll}
\hline \multicolumn{2}{l}{ 凝结时间/min } & \multicolumn{2}{l}{ 抗压强度/MPa } & \multicolumn{2}{l}{ 抗折强度/MPa } \\
\hline 初凝 & 终凝 & $3 \mathrm{~d}$ & $28 \mathrm{~d}$ & $3 \mathrm{~d}$ & $28 \mathrm{~d}$ \\
105 & 190 & 27.0 & 55.1 & 7.1 & 8.4 \\
\hline
\end{tabular}

(1)水泥: 强度等级 42.5 的普通硅酸盐水泥产自唐山冀 东水泥股份有限公司, 其主要性能如表1所示;

(2)乙二醇: 分析纯试剂;

(3)羟丙基甲基纤维素醚（表2中简称 “HPMC”）：粘 度为 $75000 \mathrm{mp} \cdot \mathrm{s}$;

(4)减水剂: SUNBO PC-1016 (P) 聚羧酸系减水剂;

(5)硅灰: 比表面积为 $39200 \mathrm{~m}^{2} / \mathrm{kg}$;

(6)玻璃纤维: 长度 $9 \mathrm{~mm}$, 直径 $14 \mu \mathrm{m}$;

(7) 早强剂: 液态 160 早强剂, 主要成分为 $\mathrm{Al}_{2}\left(\mathrm{SO}_{4}\right)_{3}$;

(8) $\mathrm{SiO}_{2}$ 气凝胶: 韩国进口, 其傅利叶红外光谱 (FT-IR) 及X-Ray衍射图谱 (XRD) 如图1、图2所示; 从图1可以看 出, $1130 \mathrm{~cm}^{-1}$ 处强而宽的吸收带是 $\mathrm{Si}-0-\mathrm{Si}$ 反对称伸缩振 动, $760 \mathrm{~cm}^{-1} 、 456 \mathrm{~cm}^{-1}$ 处的峰为 $\mathrm{Si}-0$ 键对称伸缩振动和弯 曲振动, $2963 \mathrm{~cm}^{-1}$ 和 $2907 \mathrm{~cm}^{-1}$ 处是 $-\mathrm{CH}_{3}$ 中 C-H的对称伸缩 振动和不对称伸缩振动, $1387 \mathrm{~cm}^{-1}$ 和 $760 \mathrm{~cm}^{-1}$ 是 $-\mathrm{CH}_{3}$ 中 C-H 的剪式弯曲振动和平面摇摆弯曲振动, $1256 \mathrm{~cm}^{-1}$ 和 $851 \mathrm{~cm}^{-1}$ 是 $\mathrm{Si}-\mathrm{C}$ 的振动峰, 说明在 $\mathrm{SiO}_{2}$ 气凝胶的末端支链上连有疏 水基团 $-\mathrm{CH}_{3}, 972 \mathrm{~cm}^{-1}$ 处的峰是 $\mathrm{Si}-\mathrm{OH}$ 弯曲振动峰, 说明该 气凝胶支链末端仍有亲水性基团- $\mathrm{OH}$ 存在, $3400 \mathrm{~cm}^{-1}$ 处宽 而不明显的谱带及 $1633 \mathrm{~cm}^{-1}$ 处的峰分别是 $\mathrm{H}-\mathrm{O}-\mathrm{H}$ 的不对称 伸缩振动和弯曲振动峰, 说明该样品放置空气中易吸附水 分, $3400 \mathrm{~cm}^{-1}$ 处极不明显, 表明吸附空气中水分较少 [4-5]。
图2内有二个馒头状的峰, 衍射峰主峰约在 $22^{\circ}(2 \theta)$, 与 无定形 $\mathrm{SiO}_{2}$ (如石英玻璃和硅胶等 ${ }^{[6]}$ ) 的主峰相当, 由此可 见, $\mathrm{SiO}_{2}$ 气凝胶的结晶程度很差, 为无定形 (非晶态) 的 物质。

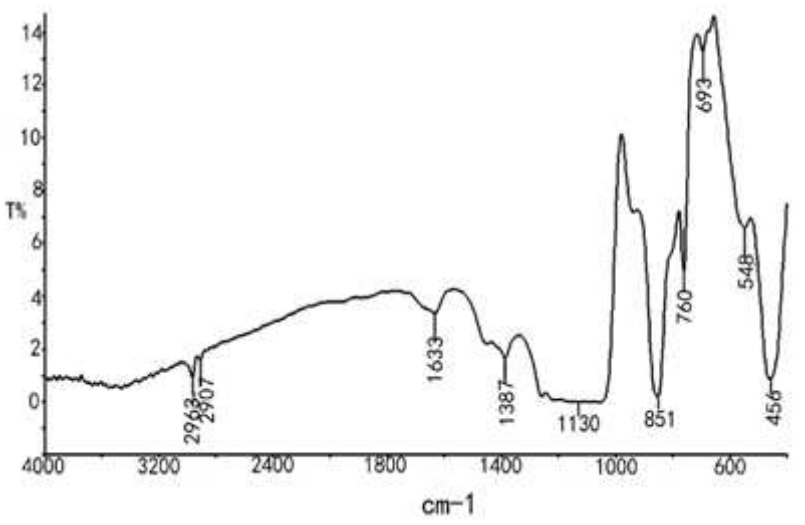

图1 气凝胶FT-IR图谱。

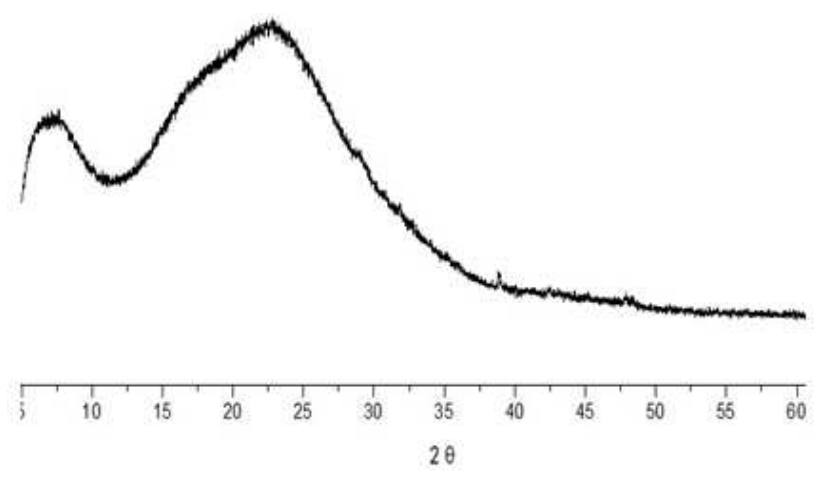

图2 气凝胶XRD图谱。

\section{3. 试验过程}

\section{1. 试件制作}

将水泥及其他粉料（硅灰, 减水剂, 玻璃纤维, 早强 剂等) 预拌 $2 \mathrm{~min}$, 再加入水和早强剂进行摚拌, 并控制新 拌料浆的初始状态, 这一过程一般搅拌 $1^{\sim} 2 \mathrm{~min}$, 随后在高 速搅拌的同时, 迅速加入化学发泡剂, 继续摚拌约 $6{ }^{\sim} 8 \mathrm{~s}$, 最终将摚拌均匀的料浆倒入模具内静停发泡, 待试件达一 定强度后拆模, 所有试件均采用自然养护至 $28 \mathrm{~d}$ 时进行各 项性能测试。

气凝胶的掺入方式分二种: (1)将气凝胶直接与水泥等 粉料混合后加水搅拌制作成型（以下简称 “直掺法” ); (2)将气凝胶先与乙二醇混合成悬浮状态, 再将其与羟丙基 甲基纤维素醚 (以下简称 “纤维素醚”) 一起掺入水泥等 粉料中进行搅拌（以下简称 “预混法” ）。配合比及其性 能见表2。

\section{2. 性能测试方法及主要仪器}

抗压强度按GB/T 5486-2008《无机硬质绝热制品试验 方法》测定, 体积吸水率按 JG/T 041-2011《复合发泡水 
泥板外墙外保温系统应用技术规程》附录A测定，导热系 数按 $\mathrm{GB} / \mathrm{T} 10294-2008$ 《绝热材料稳态热阻及有关特性的测 定防护热板法》测定, X-衍射图谱（XRD）采用德国布鲁 克AXS有限公司产D8X射线衍射仪测试, 傅立叶红外光谱 (FT-IR) 采用PERKIN-ELMER产PE1750傅立叶变换红外光 谱仪测试。扫描电镜图 (SEM) 采用日本日立公司的S-3400N 型的扫描电镜测试。
龄期 $28 d$ 时的试件取样进行FT-IR、XRD和SEM测试, 其 FT-IR图谱见图3, XRD图谱见图4, SEM图见图5、图6。

\section{4. 结果与分析}

表2. 气凝胶的掺入对发泡混凝土性能的影响。

\begin{tabular}{|c|c|c|c|c|c|c|c|c|}
\hline 编 & $\begin{array}{l}\text { 气凝胶掺入 } \\
\text { 率 } / \% \\
\end{array}$ & $\begin{array}{l}\text { 普通硅酸 } \\
\text { 盐水泥/g }\end{array}$ & $\begin{array}{l}\mathrm{SiO}_{2} \text { 气凝 } \\
\text { 胶 } / \mathrm{g}\end{array}$ & $\begin{array}{l}\text { 羟丙基甲基纤维 } \\
\text { 素醚/g }\end{array}$ & 乙二醇 /g & 体积吸水率 $/ \%$ & 28d抗压强度/MPa & 导热系数/W/ (m k) \\
\hline $\mathrm{A} 1$ & 0.0 & 800 & - & \multirow{6}{*}{ - } & \multirow{6}{*}{-} & 8.0 & 0.521 & 0.079 \\
\hline A2 & 0.5 & 796 & 4 & & & 7. 4 & 0.472 & 0.072 \\
\hline A3 & 1.0 & 792 & 8 & & & 7.2 & 0.419 & 0.074 \\
\hline A4 & 1.5 & 788 & 12 & & & 6.9 & 0.401 & 0.068 \\
\hline A5 & 2.0 & 784 & 16 & & & 6.4 & 0.357 & 0.062 \\
\hline A6 & 2.5 & 780 & 20 & & & 6.0 & 0.329 & 0.060 \\
\hline B1 & 0.0 & 800 & - & \multirow{6}{*}{0.18} & \multirow{6}{*}{16} & 7.6 & 0.535 & 0.079 \\
\hline B2 & 0.5 & 796 & 4 & & & 7.2 & 0.480 & 0.073 \\
\hline B3 & 1.0 & 792 & 8 & & & 6.7 & 0.445 & 0.073 \\
\hline B4 & 1.5 & 788 & 12 & & & 6.3 & 0.423 & 0.066 \\
\hline B5 & 2.0 & 784 & 16 & & & 5.9 & 0.402 & 0.055 \\
\hline B6 & 2.5 & 780 & 20 & & & 5.5 & 0.375 & 0.051 \\
\hline
\end{tabular}

\section{1. 气凝胶的掺入对发泡混凝土抗压强度的影响}

从表 2 可以看出, 无论是直掺法还是预混法, 气凝胶 的掺入均会使混凝土强度下降, 且随其掺量的增加, 强度 下降幅度增大, 这一方面是因为随气凝胶掺量增加水泥含 量相对下降, 另一方面也与气凝胶自身强度低有关, 气凝 胶虽然掺量不高, 但其占居的体积较大, 自身强度又低, 相当于增加了发泡混凝土固体相的孔隙率, 因而强度降低。 但当气凝胶掺量相同时, 预混法发泡混凝土强度较直掺法 略高, 且随气凝胶掺量的增加其强度下降幅度较直掺法小。

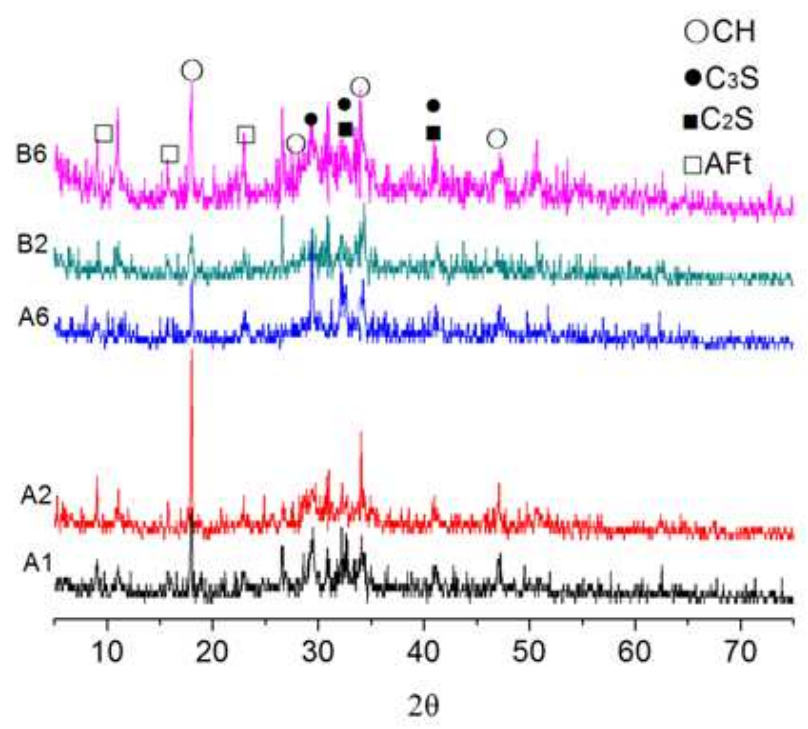

图3 气凝胶不同掺量时发泡混凝土XRD图谱。
从图 3 可以看出, 直掺法掺入 $0.5 \%$ 气凝胶时 (图 3 中 $A 2$ ), 与不掺气凝胶相比 (图3中A1) , $\mathrm{Ca}(\mathrm{OH})_{2}$ 晶体 (以下简称 “ $\mathrm{CH}$ ” ) 的 (001) 面 ( $\mathrm{d}=0.490 \mathrm{~nm})$ 的衍射峰显著增强, 而 (101) 面 ( $d=0.263 \mathrm{~nm}$ ) 衍射峰减弱, 即其晶体取向 性增强, 取向指数达到2.77 ( $\mathrm{CH}$ 晶体以 (101) 晶面为参照 面时, 其 $(001)$ 晶面的取向指数 $\mathrm{R}=\left(\mathrm{I}_{(001)} / \mathrm{I}_{(101)}\right) / 0.74$ 。若 $\mathrm{CH}$ 晶体无取向时, $\mathrm{R}=1$; 有取向时, $\mathrm{R}>1$ ) [7]。图4 (A2) FT-IR 图谱中 $3647 \mathrm{~cm}^{-1}$ 处CH的特征峰较不掺时更加明显, 表明少 量加入气凝胶时, 其高表面活性 [8-9] 早期对水泥水化有 促进作用 [10], 使之深度水化, 生成更多的 $\mathrm{CH}$, 同时, 由 于发泡混凝土的多孔性, 使生成的 $\mathrm{CH}$ 有较大的空间发生取 向性排列, 但由于气凝胶掺量少不足以发生火山灰反应而 消耗 $\mathrm{CH}$, 加之生成的 $\mathrm{CH}$ 取向度高以及水泥用量的相对减少, 因而强度产生一定程度的下降。

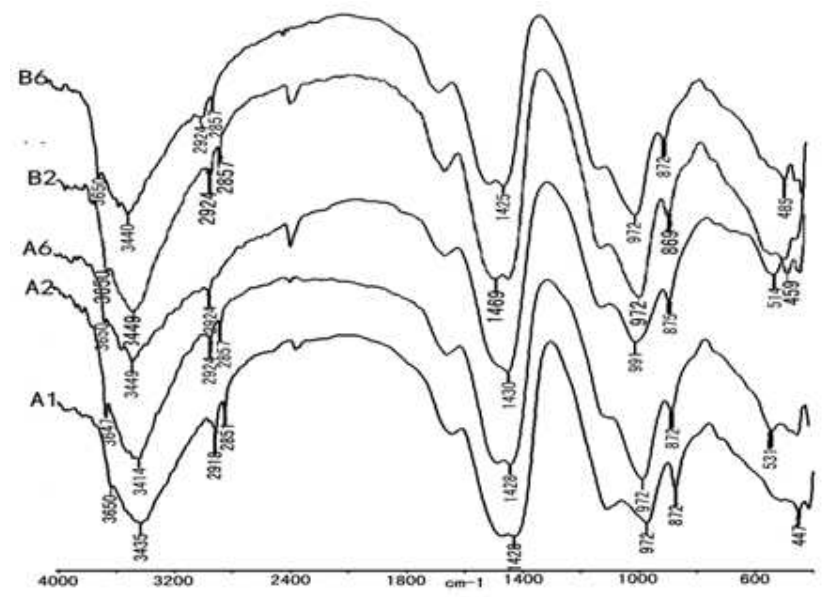

图4 气凝胶不同掺量时发泡混凝土FT-IR图谱。 
图5为直掺气凝胶的发泡混凝土SEM图, 从中可以看出, 体系中六方 $\mathrm{CH}$ 晶型完整清晰, 且呈散落状, 与周围的钙矾 石等物相边界清楚、相互结合程度低, 散落的 $\mathrm{CH}$ 取向程度 较大。

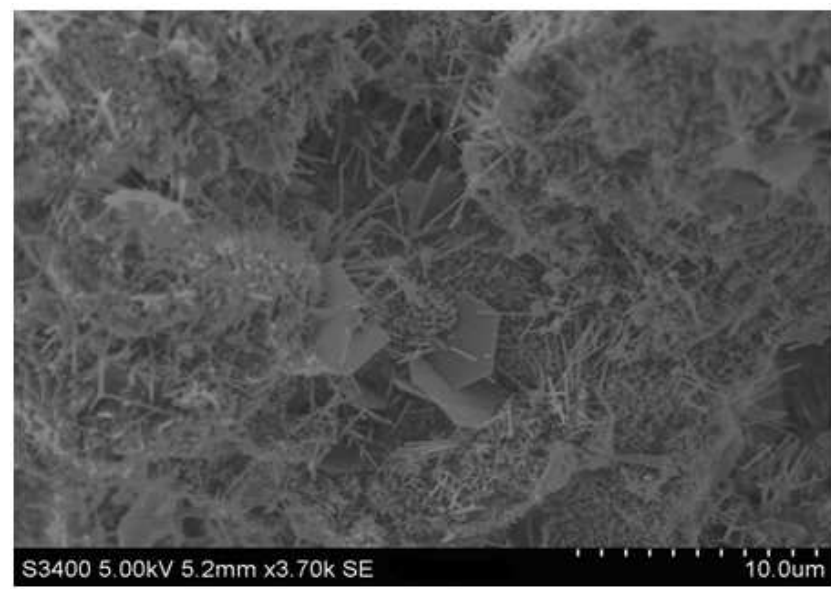

图5 未掺纤维素醚的发泡混凝土SEM图。

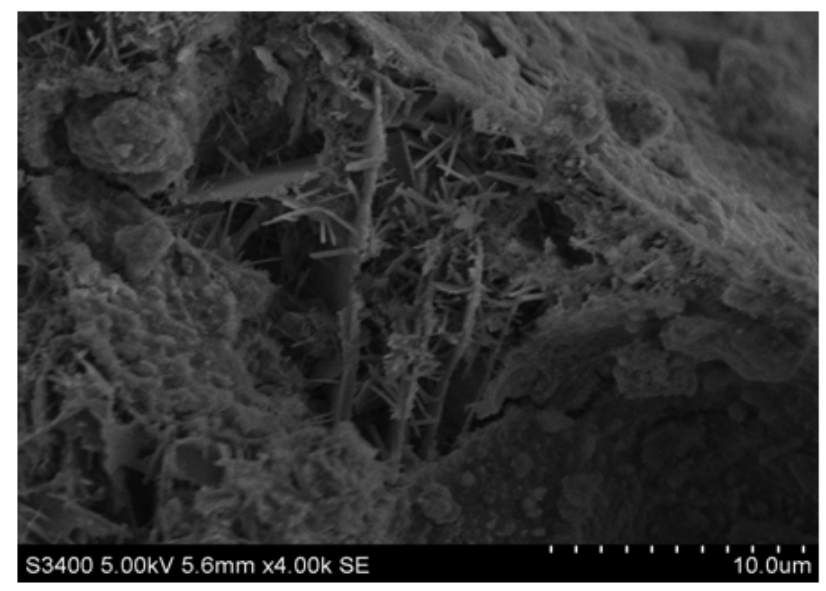

图6 掺加0.05\%纤维素醚后的发泡混凝土SEM图。

当直掺法掺入 $2.5 \%$ 气凝胶 (图3中A6) 时, $\mathrm{CH}(001)$ 面的衍射峰较掺入 $0.5 \%$ 时明显减弱, 且取向程度也明显下 降 (取向指数下降到1.12)，图4（A6）中 $3650 \mathrm{~cm}^{-1}$ 处 $\mathrm{CH}$ 的特征峰也减弱, 但 $\mathrm{C}-\mathrm{S}-\mathrm{H}$ 的特征峰左移, 从 $972 \mathrm{~cm}^{-1}$ 移到 了 $991 \mathrm{~cm}^{-1}$, 这是 $\mathrm{C}-\mathrm{S}-\mathrm{H}$ 聚合度增加的表现, 这说明增加气 凝胶的掺量后, 高活性的气凝胶中的 $\mathrm{Si}-\mathrm{O}-\mathrm{Si}$ 键在碱性环 境中断裂, 形成的水化硅酸根单体与 $\mathrm{C}-\mathrm{S}-\mathrm{H}$ 及 $\mathrm{CH}$ 解离的 $\mathrm{Ca}^{2+}$ 和 $\mathrm{H}^{-}$结合, 形成高聚 $\mathrm{C}-\mathrm{S}-\mathrm{H}$, 既消耗了体系中的 $\mathrm{CH又}$ 提高 了 $\mathrm{C}-\mathrm{S}-\mathrm{H}$ 的聚合度 [11-12], 这有利于发泡混凝土强度的提 高; 但另一方面, 当气凝胶掺量多时, 由于气凝胶粒子比 表面积巨大、存在不饱和残键和不同键合状态的羟基 [13], 与水泥水化产生的 $\mathrm{Ca}^{2+} 、 \mathrm{SO}_{4}{ }^{2+} 、 \mathrm{Na}^{+} 、 \mathrm{OH}^{-}$等离子结合, 不仅 导致气凝胶粒子本身团聚, 且将水泥颗粒团聚在一起, 妨 碍水泥颗粒水化 [14], 因此与掺入 $0.5 \%$ 比, 气凝胶掺量 $2.5 \%$ 时, 团聚作用增大, 水泥水化也受影响 (图3中A6表现为 熟料矿物衍射峰增强）, 因此, 虽然有部分气凝胶发生二 次水化反应, 仍不足以弥补由于水泥用量减少、水泥水化 受阻以及气凝胶团聚作用带来的强度的降低。
预混法气凝胶掺量 $0.5 \%$ 时与直掺法气凝胶掺量 $0.5 \%$ 时相比, 图3 (B2) 可明显看出, CH的 (001) 面衍射峰 减弱, 且取向程度大大降低（取向指数仅为 $0.74 ）$, 而 熟料矿物的特征峰较直掺 $0.5 \%$ 时增强, 图4 (B2) 中 3640 $\mathrm{cm}-1$ 处 $\mathrm{CH}$ 的特征峰也减弱, 原因有两个方面: (1)当掺入 纤维素醚时, 纤维素醚可快速与水分子形成氢键并溶解 在水中 [15], 一方面增加了水泥浆体的粘度, 导致 $\mathrm{Ca}^{2+}$ 、 $\mathrm{SO}_{4}{ }^{2+} 、 \mathrm{OH}^{-}$等离子的移动速率降低, 另一方面, 纤维素 醚吸附在未水化的水泥颗粒和水化产物的表面, 阻止了 水泥颗粒与水接触进行反应及水化产物成核、长大和沉 淀 [16], 两种效应共同作用, 延缓了水泥的水化, 因而 表现出CH生成量少、取向度低。(2)由于气凝胶本身具有 多孔材料的物理吸附性 [17-20], 其硅羟基又易与纤维素 醚上的羟丙基结合而吸附于纤维素醚之上 [21], 这样, 纤维素醚既相当于 “桥架”, 又相当于 “隔离剂”, 阻 碍了气凝胶与水泥的接触反应, 从而阻止或阻碍了其直 掺时对水泥水化的促进作用。两个方面原因共同作用下， 使得预混法气凝胶掺量 $0.5 \%$ 与直掺法气凝胶掺量 $0.5 \%$ 时 相比, 强度变化不大。

当预混法气凝胶掺量 $2.5 \%$ 时, 与预混法气凝胶掺量 $0.5 \%$ 相比, 图3 (B6) 中CH (001) 面的特征峰强度和取向 度增强（取向指数达到1.55）, 图4（B6）中 $3650 \mathrm{~cm}-1$ 处CH的特征峰也略有增强, 这是因为, 纤维素醚含量不变, 而增加气凝胶掺量时, 其效果类似于直掺 $0.5 \%$ 时, 一部分 未被纤维醚 “桥架阻隔” 的气凝胶仍会对水泥的深度水化 有促进作用, 但由于气凝胶掺量增加, 相当于增加了发泡 混凝土固体相的孔隙率，同时水泥掺量减少，加之CH取向 程度增加, 使之较预混0.5\%时强度下降。但与直掺 $2.5 \%$ 时比, 强度下降幅度要小, 从图3 (B6) 可见, $\mathrm{CH}$ 产物增 多, 且取向度增加, 而熟料矿物相减少, 说明纤维素醚与 气凝胶结合的 “隔离剂” 作用不仅减少了气凝胶的团聚作 用, 也减小了气凝胶因与水泥水化产物作用而使水泥团聚 的作用, “解放” 了一部分气凝胶, 一方面有利于气凝胶 与水泥水化产物中的 $\mathrm{CH}$ 发生二次水化反应, 另一方面也恢 复了水泥的正常水化作用，同时，这种 “桥架” 作用也更 有利于发泡混凝土固体相中的物质连接成一个整体。图6 为预混法掺入气凝胶的发泡混凝土的SEM图, 与图5相比可 看出, 固体相中 $\mathrm{CH} 、 \mathrm{AFt}$ 及 $\mathrm{C}-\mathrm{S}-\mathrm{H}$ 凝胶等物相上面有覆盖物 (为纤维素醚), 且边界模糊, 结合紧密。综合以上因素, 因此强度较直掺法气凝胶掺量 $2.5 \%$ 时下降幅度减小。

\section{2. 气凝胶的掺入对发泡混凝土导热系数的影响}

在发泡混凝土中掺入气凝胶降低其导热系数的理想 状态是保证气凝胶在发泡混凝土中的完整性、分散性及 与发泡混凝土本体的粘结性, 即, 气凝胶本身的纳米孔 结构不因掺入发泡混凝土体系中而被破坏、气凝胶颗粒 能单颗粒分散在发泡混凝土中而不团聚、气凝胶颗粒能 与泡沫混凝土本体结合成一个整体而不脱出。而实际上, 气凝胶因具有以下特点而很难达到以上理想状态: (1)强 度低、脆性大, 纳米级孔隙结构在外在压力（搅拌、成 型）下很容易被破坏，(2)其表面开放多孔结构以及亲水 性基团硅羟基的存在使之非常容易吸收水或水蒸气，造 
成材料的载荷大量增加, 抗拉强度大幅度降低, 有时甚 至会导致气凝胶开裂直至完全损坏，(3)由于硅气凝胶的 主要成份是 $\mathrm{Si}_{2}$, 极易与水泥的水化产物中的 $\mathrm{CH}$ 等成份 发生化学反应而破坏其自身的结构, (4)纳米级的 $\mathrm{SiO}_{2}$ 也 极易团聚，(5)气凝胶颗粒极轻，在掺入发泡混凝土中时 容易逸散。这些因素, 均可使气凝胶对发泡混凝土绝热 性能的改善效果产生不利影响。

从表 2 可以看出, 无论是直掺法还是预混法, 随着气 凝胶掺量的增加, 对发泡混凝土的导热系数确实产生了改 善作用，但掺入方式不同，改善效果有异。直掺时，导热 系数从 $0.079 \mathrm{~W} /(\mathrm{K} \cdot \mathrm{m})$ 降低到了 $0.06 \mathrm{~W} /(\mathrm{K} \cdot \mathrm{m})$ ，降 低了 $24 \%$, 而预混时, 导热系数从 $0.079 \mathrm{~W} /(\mathrm{K} \cdot \mathrm{m})$ 降低 到 $0.051 \mathrm{~W} /(\mathrm{K} \cdot \mathrm{m})$ ，降低了 $35 \%$ 。

气凝胶直掺时, 掺量从 $0.5 \%$ 增加到 $2.5 \%$ 时, 导热系数 一定程度下降, 这是由于随着气凝胶掺量的增加水泥用量 相对减少，同时气凝胶的掺入相当于增加了发泡混凝土固 体相的孔隙率, 同时当其占居发泡混凝土原有的孔隙时, 相当于减小了其孔径并将开口孔变成了闭口孔, 因而导热 系数下降, 但下降幅度较预混法小。

预混法掺入气凝胶时, 随气凝胶掺量增加导热系数 下降程度较之直掺法大, 特别是气凝胶掺量 $2.5 \%$ 时, 纤 维素醚的作用对导热系数的减少有较明显的作用, 原因 为: (1)当气凝胶直掺且掺量大时, 虽然其本身会团聚, 但总有部分气凝胶不可避免的与水泥水化产物中的 $\mathrm{CH}$ 发 生火山灰反应使其本身的纳米孔结构遭到破坏, 而当掺 入纤维素醚时, 其 “桥梁” 或 “隔离剂” 的作用一定程 度的阻碍了气凝胶与水泥的反应, 即, 预混法有利于保 持气凝胶的完整性, 从而提高气凝胶在发泡混凝土试件 中的有效利用率。(2)由于纤维素醚对水分通过氢键的吸 附作用, 也减少了气凝胶与水直接接触而破坏其自身结 构的几率。(3)纤维素醚对水泥浆体的增稠作用也有利于 搅拌时气凝胶材料的分散, 同时对浆体的增稠也有利于 气孔的稳定存在。这些因素均对导热系数的下降有促进 作用。因而预混法与直掺法比, 在气凝胶掺量相同时, 其导热系数改善效果要好。

\section{3. 气凝胶的掺入对发泡混凝土体积吸水率影响}

从表 2 可以看出, 随着气凝胶掺量的增加, 体积吸水 率下降。直掺法吸水率下降了 $25 \%$, 而预混法降低了 $35 \%$ 。 原因是虽然气凝胶的掺入相当于增加了发泡混凝土的孔 隙率, 但由于气凝胶纳米级的闭口结构, 实质上是改善了 发泡混凝土的孔结构, 即将部分开口孔变成了闭口孔, 同 时纲米级的气凝胶也相当于使发泡混凝土的孔径变小, 这 些因素均有利于降低其吸水率; 另一方面, 气凝胶颗粒比 表面积大, 需水量大, 因而随气凝胶掺量的增加浆体流动 度下降, 有助于泡沫的稳定, 减少了泡沫破裂和开口孔增 加的几率。

预混法较直掺法吸水率更小, 原因是纤维素醚的 “桥 架” 作用能减少气凝胶因与水泥发生火山灰反应而产生的 破坏作用, 保持气凝胶纳米闭孔的完整性, 另一方面, 其 保水作用也能够减少发泡混凝土因失水产生的连通孔的 几率, 这些因素都会减小其体积吸水率。

\section{5. 结论}

1) 气凝胶直接掺入发泡混凝土中对其性能的改善作 用有限，掺量 $0.5 \%$ 时导热系数改善效果甚微，掺量 $2.5 \%$ 时导热系数虽有改善但抗压强度下降程度达 $36.8 \%$, 原因 是掺量少时有利于促进水泥水化, 但会增加 $\mathrm{CH}$ 取向性; 掺 量多时又会产生团聚, 均不利于发泡混凝土性能的改善。

2）将气凝胶与乙二醇和羟丙基甲基纤维素醚预混后 再掺入泡沫混凝中, 可改善发泡混凝土绝热性能、降低吸 水率, 气凝胶掺量 $2.5 \%$ 时可使导热系数降低 $35 \%$ 、体积吸 水率降低 $31 \%$, 且抗压强度达到 $0.375 \mathrm{MPa}$, 下降幅度减小 $29.5 \%$, 非常接近 JG/T041-2011《复合发泡水泥板外墙外 保温系统应用技术规程》中 $0.40 \mathrm{MPa}$ 的要求, 原因是羟丙 基甲基纤维素醚有利于保持气凝胶的完整性, 即预混法可 提高气凝胶在发泡混凝土中的掺量, 从而达到改善其绝热 效果的目的。

3）预混法气凝胶掺量 $2.5 \%$ 时, 抗压强度偏低, 仅有 $0.375 \mathrm{MPa}$, 已将气凝胶、早强剂、膨胀剂、硅灰为研究因 素进行了正交试验, 可在保持导热系数 $0.051 \mathrm{~W} /(\mathrm{m} \cdot \mathrm{k})$ 的情况下使抗压强度提高到 $0.640 \mathrm{MPa}$, 篇幅所限, 后续 将另行撰文阐述。

\section{参考文献}

［1］中华人民共和国国家发展和改革委员会. JC/T 1062-2007. 发泡混凝土砌块 $[\mathrm{S}]$. 北京: 中国建材工业出版社, 2008。

［2］中华人民共和国住房和城乡建设部. JG/T314-2012. 聚氨酯 硬泡保温板 [S]. 北京: 中国标准出版社, 2012。

［3］中华人民共和国建设部. JG149-2003 膨胀聚苯板薄抹灰 外墙外保温系统 $[\mathrm{S}]$. 北京: 中国标准出版社, 2003。

[4] EL RASSY H, PIERRE A.C. NMR and IR spectroscopy of silica aerogels with different hydrophobic characteristics [J] (Eng). Journal of Non-Crystalline Solids. 2005, 351(7): $1603-1610$.

[5] RAMI AL-OWEINI, HOUSSAM EL-RASSY. Synthesis and characterization by FTIR spectroscopy of silica aerogels prepared using several $\mathrm{Si}(\mathrm{OR}) 4$ and $\mathrm{R}^{\prime}$ ' $\mathrm{Si}\left(\mathrm{OR}{ }^{\prime}\right.$ ) 3 precursors [J] (Eng). Journal of Molecular Structure. 2009, $919(2): \quad 140-$ 145http://www. sciencedirect. com/science/article/pii/S 0022286008005759 - cor1mailto:Houssam. Rassy@aub. edu. 1b

[6] 浙江大学, 等. 硅酸盐物理化学 [M]. 北京: 中国建筑工业出 社, 1980.120。

[7] 叶青 硅溶胶对水泥基材料微观结构和力学性能的影响 [J] 硅酸盐学报2008，36（4）：425-430。

[8] ZAVODINSKY V G, KUYANOV I A, ZAVODINSKAYA 0 M. Atomic and electronic structures of nanometer sized silica particles[J] (Eng). Journal of Non-Crystalline Solids. 1999, $243(2-3): 123-136$. 
[9] 徐子芳, 王君, 张明旭. 纳米级Si02改性水泥胶砂作用机理 研究 $[J]$. 硅酸盐通报, 2007, 26 (1)：58-63。

[10] 范基骏, 从立庆, 陈荣民. 探讨水泥基纳米复合材料的纳米 诱导水化理论 [J]. 建材发展导向, 2005 (4) : 37-44。

[11] 王䂞, 何真, 杨华全等. 硅粉增强混凝土抗冲磨性能的微观 机理 $[J]$. 水利学报, 2013, 44(1)：111-118。

[12] 杨南如, 岳文海. 无机非金属材料图谱手册 [M] 武汉 武汉 工业大学出版社，2000：337-338。

[13] 吴国友, 程璇, 余显尔等常压干燥制备二氧化硅气凝胶 [J]. 化学进展, 2010, 22 (10) : 1892-1900。

[14] 孔德玉, 杜祥飞, 杨杨等. 纳米二氧化硅团聚特性对水泥水 化硬化性能的影响 [J]. 硅酸盐学报，2012，40 (11) : 1599-1606。

[15] 苏雷, 马保国, 寒守卫等. 纤维素醚-珍珠岩耦合作用下水 泥浆体早期水化规律 $[\mathrm{J}]$. 功能材料, 2012，43（15）： 2102-2105。
[16] 张国防, 王培铭. 羟乙基甲基纤维素影响水泥浆体微观结构 的研究 $[\mathrm{C}] / /$ 第三届全国商品砂浆学术会议, 武汉, 2009: 150-157。

[17] 曾永, 刘敬肖, 史非二氧化硅气凝胶对硫酸庆大霉素的吸 附和释放 [J]. 硅酸盐学报, 2007, 35 (8)：1081-1085。

[18］张志华, 倪星元, 陈世文等 Si02气凝胶常压制备、表面结构 与吸附性质 $[J]$. 原子能科学技术, 2005, 39 (6) : 498-501。

[19] 吴俊升, 李晓刚, 杜伟等 纳米多孔气凝胶材料在催化和吸 附领域的应用 [J]. 功能材料, 2004，35（7）:2682-2688。

[20] SOLEIMANI DORCHEH A, ABBASI M. H. Silica aerogel; synthesis, properties and characterization[J] (Eng). Journal of materials processing technology. 2008, 199:10-26.

[21] 邬润德, 童篖莉, 王锐兰. 聚丙烯酸酯原位乳液聚合包覆纳 米硅溶胶的研究 [J]. 有机硅材料, 2002, 16 (5) :5-8。 